\title{
Happiness: Role of Dopamine and Serotonin on Mood and Negative Emotions
}

Elena Baixauli*

Department of Psychology, University of Valencia, Spain

*Corresponding author: Elena Baixauli, Department of Psychology, University of Valencia, Spain, Tel: +3496386 4100; E-mail: elena.baixauli@uv.es

Received date: March 24, 2017; Accepted date: April 13, 2017; Published date: April 20, 2017

Copyright: (c) 2017 Baixauli E. This is an open-access article distributed under the terms of the Creative Commons Attribution License; which permits unrestricted use; distribution; and reproduction in any medium; provided the original author and source are credited.

\begin{abstract}
Dopamine is a hormone associated with happiness and serotonin regulates our mood. When a person is physically attracted to another, an activation of dopamine, serotonin increased and production of oxytocin, a hormone that reduces pain perception and increases the emotional connection we have with the other occurs. Disconnecting the amygdala, the parts of the brain that are active against negative emotions like fear. The combination of all these elements leads to crush the other. After our beliefs, the way we understand ourselves and understand the world around us, eventually determining if I made a good choice. These are questions necessary to understand the functioning of our brain and human relations. Maybe if we are able to answer these questions we can prevent many interpersonal conflicts. All to indicate that it is not, perhaps a cultural thing, something we have learned. I mean, throughout history we have learned that the best defence is attack and then our amygdala is always on, then it is easier to move quickly to negative emotions.
\end{abstract}

Keywords: Dopamine; Oxytocin; Dysfunctional interactions; Emotions

\section{Introduction}

Many times we get up and go to bed, get up and go to bed.

What is the meaning of life? Finding an answer is not easy when you see the suffering and pain of people around you, the damage we do to each other.

What is the meaning of life? In a dehumanized world, full of emotions and devoid of feelings.

The meaning of life is that engine that fills the soul. It is your true essence; it is your freedom that you can never take away, our capacity to be.

Dysfunctional interactions between the serotonin and dopamine systems in the prefrontal cortex can lead to aggression. Specifically, serotonin hypofunction may represent a biochemical trait that predisposes individuals to aggression [1].

Recalled sadness was associated with increased activation in the anterior insula. Happiness was distinguished from sadness by greater activity in the vicinity of ventral mesial frontal cortex Lane [2].

Mild changes in negative mood appear to have little effect on cognitive control processes, while positive mood affects aspects of actualization, planning, and change. These cognitive effects may be related to neurochemistry: with positive mood effects mediated by dopamine, while the negative effects of mood may be mediated by serotonin levels. Current evidence on the effects of mood on regional brain activity during executive functions indicates that the prefrontal cortex is a recurrent site of integration between mood and cognition [3].
The Parisians knew about it, so they sang their hymn in the slaughter of the neighboring country, no one can take away their identity!

Every person needs to find the meaning of life, since it makes us dream, sings, laugh, cry and make mistakes to learn. Without this sense, we wander the world, but we do not live.

The meaning of life is to know why you are here, what you can bring.

In most of the conflicts we have with others we end up thinking that if we have problems it is for them, so we dedicate our existence to making life impossible to those around us. Perhaps because they are different from us, because they think differently, without stopping to think that the good thing is that we are unique beings [4-7].

We live more aware of the other, of pleasing, of being accepted socially, of causing harm, of being the centre of attention, of having an eagerness to be protagonist, of being recognized each and every one of our achievements, which we live as the poet "I live without living in me".

The search for the meaning of life leads us to the "I", to a reflection on who we are and what we want to do, distances us from the interpersonal conflict, gives meaning to our existence, marks our objectives, step by step, step by step, climb the ladder of our dreams, our goals, what we want to achieve, that makes us feel happier.

When you find the meaning of life you find peace, because you feel satisfied and learn that only by being well with yourself, you can be well with others.

And we change our language, we no longer say "it's you", we say "I thank you".

And you think it's good, to feel accompanied in this active quest to dream, think, feel and love.

Look for your sense of life! 
I found him, the day I decided: what the meaning of my life was to help others find theirs!

There are people who feel incomplete and seek in others to fill that existential emptiness.

They are people who undertake many things, travel, know new cultures, perform multiple activities, have several relationships, but do not feel that nothing fills them.

They are people who seek the acceptance of others, stop being they to become what they believe they should be, in their concern to be how others want, for fear of rejection, to never be able to cover the void they feel.

Many times we are not aware of the importance that our past has for our existence.

The way we relate today, how we see ourselves is directly related to the affection that we received during our childhood.

\section{The Attachment Theory}

We speak of the Theory of Attachment, and from the moment the baby is born, all the emotions of the mother, as well as the actions she performs are conditioning the child's personality. When it is born, it is essential that the child receives the attention and affection necessary for his or her adaptive development.

If the mother is unable to meet the needs of the child, the child can learn not to express emotions or only express negative ones.

The Attachment Theory explains in many cases why interpersonal conflicts occur.

The affective bonds that we establish with our mother and then with our father in childhood determines to a great extent our self-concept and self-esteem.

The care that the baby receives can produce stress when the bond that stable with his mother is not safe, that is to say the mother does not affectively attend the child when it demands it. Subsequently negative or positive messages transmitted by parents to the child, not only influence their temperament but also in self-concept and selfesteem.

Those children who have not had a positive and secure emotional bond with the mother are later in adulthood people with great difficulties expressing emotions [8].

People who did not feel the affection of their parents during their childhood express more negative emotions and have more problems with their relationships.

It is easy for them to have more partner conflicts, since lack of affection in childhood prevents them from being able to positively express their emotions with the other, tend to repeat patterns of behavior. And negative affect is linked to negative thinking, a series of mental schemes, about what "should be" about life in general and in relation to others. Here too, expectations are "what I expect from others" and the value we give to things. What for one is very important for the education and the affection that has received, for the other it is not. And this incomprehension on both sides is the source of many conflicts, sometimes solvable and in others not so much.

The fact of having children sometimes increases affective problems more, since these people how they have not received that affection, they do not know how they have to express them with the others, reason why by way of modeling, the behavior is transmitted of parents to children [9].

The inability to express positive emotions increases the ability to express negative emotions that creates more conflict of relationship with others. More problems to have friends and meet new people.

Relationship problems with co-workers, because it is sometimes easy for them to think that if they treat them well, it is because they are hiding something that is going to hurt them.

The "fear of being harmed" leads them to create a kind of mask and to believe that one has to keep distances with others, only this way they can survive, it is necessary to give an image of being strong and of being able with everything.

People with insecure attachment are more prone to psychopathological problems, to being sad, to feeling alone, with low self-esteem, with dependence on others, with deficits in social skills and, of course, emotional expression.

On the contrary, those people with secure attachment, who had an affective bond with their parents, will be those people who develop greater capacity to express positive emotions and affection will be a fundamental variable in the relationship with the others.

If we consider the Theory of Attachment, we can understand the importance of working with parents in emotional intelligence as prevention of interpersonal conflicts. Here the training with the future parents is fundamental, so that from the beginning they provide the child with what he needs for his physical and psychic development.

\section{Neurological Science}

From a neurological point of view, there are hormones in our brain associated with positive emotions. Dopamine is a hormone associated with happiness and serotonin regulates our mood.

When a person is physically attracted to another, an activation of dopamine, serotonin increased and production of oxytocin, a hormone that reduces pain perception and increases the emotional connection we have with the other occurs. Disconnecting the amygdala, the part of the brain that are active against negative emotions like fear [10].

The combination of all these elements leads to crush the other. After our beliefs, the way we understand ourselves and understand the world around us, eventually determining if I made a good choice.

But what happens in our brain for these hormones of happiness, stop segregating the amounts needed?

\section{At what point is again activated the amygdala?}

These are questions necessary to understand the functioning of our brain and human relations [11].

Maybe if we are able to answer these questions we can prevent many interpersonal conflicts.

What if are training our brain? i.e., if I decide to make those things that make me happy and perform exercises to activate my serotonin and care for my electrical part of the brain, certainly my connections favour positive emotions [12].

Assuming that if I'm good I can be okay with the other.

Why is it so easy to quickly change our negative emotions? 
Citation: Baixauli E (2017) Happiness: Role of Dopamine and Serotonin on Mood and Negative Emotions. Emerg Med (Los Angel) 7: 350. doi:

Page 3 of 3

Do we have a genetic predisposition to generate negative emotions?

All to indicate that it is not, perhaps a cultural thing, something we have learned.

I mean, throughout history we have learned that the best defence is attack and then our amygdala is always on, then it is easier to move quickly to negative emotions.

This would explain why we love to hate, why we quickly forget the good times and had to focus on the bad. Our beliefs and values, our sense of self and other, help to create misinterpretations, which with the help of our brain structure will be easier to interpret as a bad response, criticism and attack.

Are we trained to generate positive emotions with others?

Tough question, if my answer is that in my life I've been happy, I will have tried to give happiness to another.

Learn to train your brain!

\section{References}

1. Michell R, Phillips L (2007) The psychological, neurochemical and functional neuroanatomical mediators of the effects of positive and negative mood on executive functions. Neuropsychologia 45: 617-629.
2. Lane RD, Reiman EM, Ahern GL, Schwartz GE, Davidson RJ (1997) Neuroanatomical correlates of happiness, sadness, and disgust. Am J Psychiatry 154: 926-933.

3. Seo D, Patrick CJ, Kennealy PJ (2008) Role of serotonin and dopamine system interactions in the neurobiology of impulsive aggression and its comorbidity with other clinical disorders. Aggress Violent Behav 13: 383-395.

4. Baixauli E (2002) The evolution of the present society: the problems of communication. Conference the Current Family: Orientation and Mediation as a Resolution of Family Conflicts, The Alcudia of Crespins.

5. Baixauli E (2002) Family mediation: A path to conflict resolution.

6. Baixauli E (2003) Community mediation and administration. European Mediation Offices, Barcelona.

7. Baixauli E (2005) To live in peace.

8. Musitu G (1995) Psychology of human communication.

9. Bandura A (1982) The theory of social learning.

10. Dorjee D (2013) Mind, brain and the path to happiness: A guide to Buddhist mind training and the neuroscience of meditation. Routledge, UK.

11. Lieberman MD (2000) Intuition: A social cognitive neuroscience approach. Psychol Bull 126: 109-137.

12. Kringelbach ML, Berridge KC (2009) Towards a functional neuroanatomy of pleasure and happiness. Trends Cogn Sci 13: 479-487. 